\section{Orthogonal-polynomial programs: Good vs. better}

\section{PHILLIP L. EMERSON \\ Cleveland State University, Cleveland, Ohio 44115}

The use of orthogonal polynomials in trend analysis with analysis of variance tests is fairly well known to psychological researchers. Grant (1956) illustrated several kinds of applications in balanced designs, and Gaito and Turner (1963) confirmed the correctness of Grant's method of choosing error terms. Gaito (1965) and others have pointed out that equal spacing and equal $\mathrm{n}$ on the trend variable are not necessary if an appropriate set of orthogonal polynomials can be constructed for the particular case of unequal spacing and weighting. Also, efficient methods of generating such polynomials for arbitrary points on the independent variable allow this technique to be used for other applications. One is general polynomial regression with correlational data, with which there may even be only one observation per value of the independent variable. Direct methods of stepwise polynomial regression require the inversion of a matrix at each step. The size of the matrix grows at each step, and the number of calculations mutliplies rapidly with each increment of the matrix size. Orthogonal polynomials can be used to combat such growth and the inaccuracies that arise from it.

Several contributions to this journal recently have reported using a certain FORTRAN subroutine (Emerson, 1965) to generate orthogonal polynomials for unequal spacing and unequal $\mathrm{n}$. The program is based on the Gram-Schmidt method and is probably adequate for the classical analysis of variance applications (linear, quadratic, and sometimes cubic components). In other applications, such as general polynomial regression, it is sometimes desirable to construct a larger set. In such cases, and even for the classical applications, one can obtain better accuracy using another method (Emerson, 1968), based on a three-term recurrence relation. The number of calculations is smaller, which partly accounts for the greater accuracy. Also, execution is faster, for the same reason. Figure 1 contains a FORTRAN subroutine using the better method. The three-term recurrence relation was used by 19 th-century mathematicians to derive quadrature formulas (Szegö, 1939/1959). Householder (1953) mentioned its numerical applications and attributed such uses to $\mathrm{C}$. Lanczos. Some program packages make use of the method and attribute it to Forsythe (1957).

The present subroutine is designed to be used in a stepwise way. The user may not know how far he wants to go until he makes some tests along the way. This is in contrast to some other programs that require a prior specification of how many orthogonal polynomials to

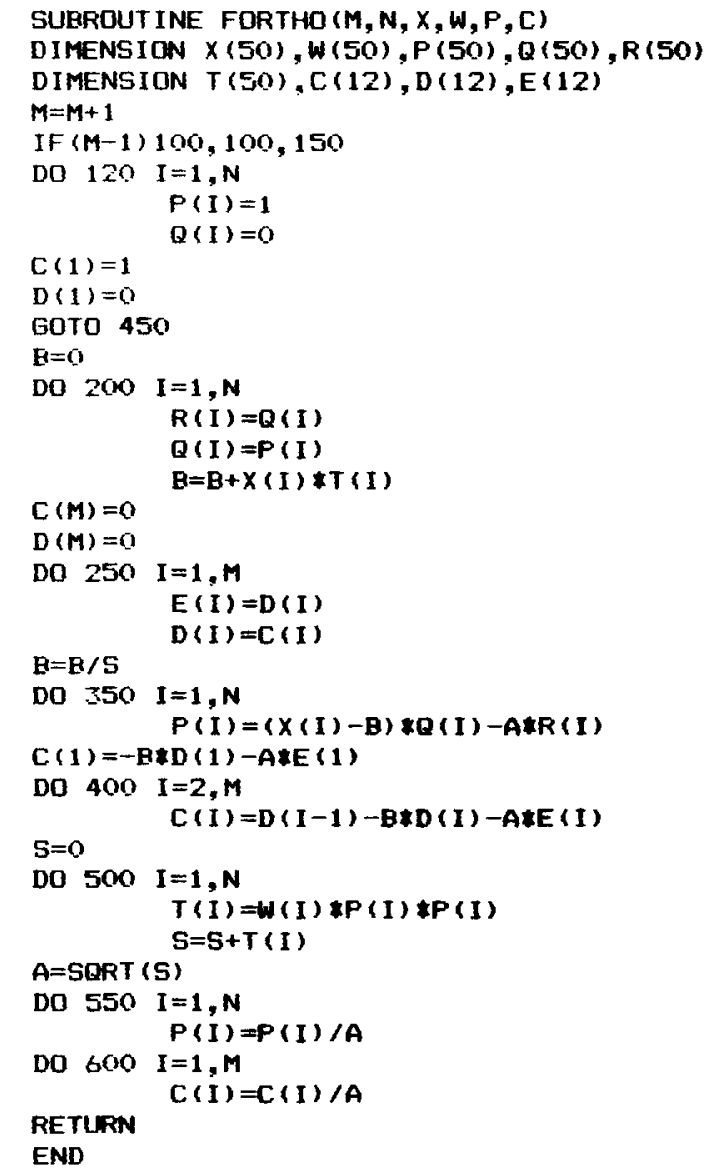

Figure 1. FORTRAN subroutine to construct orthonormal polynomials from the three-term recurrence relations, for unequal spacing and weighting. $M, N$, and $I$ are integer variables. All others are real, single or double precision. If double precision is used, the square-root function in the statement after 500 should be replaced by the double-precision square-root function.

generate. If one underestimates the number, then, there is nothing to do but start from scratch with a larger estimate. Here, the argument $M$ is used for stepping. The value of $M$ must start at 0 for the constant (degree- 0 ) polynomial, and it will proceed to 1 for the linear, 2 for the quadratic, and so on. The subroutine increments the value of $\mathrm{M}$ before returning to the calling program, each time it is called. Thus, the calling program should set $M=0$ before the first call and then simply make repeated calls to the subroutine, storing the results and perhaps performing other computations between calls. The calling program should not modify the values of $\mathbf{M}$, $\mathrm{N}, \mathrm{X}, \mathrm{W}, \mathrm{P}$, or $\mathrm{C}$ once the stepwise procedure has been started, unless it is to be started anew with $\mathbf{M}=0$. Online uses of this stepwise scheme are easily envisioned.

Before the first call, $\mathrm{N}$ should be set equal to the number of data points; the $X$ array should be filled with the $N$ values of the independent variable, and the $W$ 
array should be filled with the corresponding weights. These weights would usually be the number of observations at the corresponding levels of $\mathrm{X}$. If $\mathrm{W}$ is proportional but not equal to the numbers of observations, the proportionality factor has to be applied to the corresponding sum of squares in the analysis of variance. It cancels out in the regression coefficients, however (see Emerson, 1965, for example). Actually, the unequal weighting option is a bit redundant, since one could simply enter the same value of $X$ a number of times with $\mathrm{W}=1$ each time. In some applications, however, the user will know exactly how many distinct values of $X$ there are and how many observations there are for each distinct value. Note that the condition stated by Emerson (1965), " $\mathrm{X}(1)<\mathrm{X}(2)<\mathrm{X}(3) \ldots<\mathrm{X}(\mathrm{N})$," is entirely unnecessary for the calculations. It merely expresses the usual convention in experimental design.

The main results are returned in the $\mathbf{P}$ array for each step. This will be the orthogonal polynomial of degree $M-1$ after the call, since $M$ was incremented by the subroutine before returning. $P$ contains the values of the polynomial at the $\mathrm{N}$ corresponding values of $\mathrm{X}$. The $C$ array contains the coefficients of the powers of $X$ in the polynomial whose values are returned in the $P$ array. $\mathrm{C}(1)$ is the constant term, $\mathrm{C}(2)$ the coefficient of the linear, $\mathrm{C}(3)$ the coefficient of the quadratic, and so on, up to $C(M)$. These coefficients are not used directly in analysis of variance and the like, but they can be used to convert an orthogonal-polynomial regression equation to explicit power-series form. If these $\mathrm{C}$ coefficients will not be needed, Statements $130,140,220$, $230,250,360,370,400,570$, and 600 may be omitted from the subroutine, and so may the argument $C$ and the dimensioned variables $\mathrm{C}, \mathrm{D}$, and $\mathrm{E}$. The polynomials that are generated are orthonormal (i.e., $\Sigma W P^{2}=1$ ), and this is a convenience in some applications. The program limits $N$ to 50 and $M$ to 10 , but these can be changed merely by changing the dimension statement.
Some cautions are necessary. There is a mathematical limit to the value of $M$. It is the number of distinct values of $\mathrm{X}$, minus 1 . With this many orthogonal polynomials, the regression equation will fit the means exactly. Another caution is that in statistical calculations, computers are known to give poor results due to truncation errors, under some circumstances. A well known case is when the mean of the numbers being processed is large relative to their standard deviation. For that reason, it is a good idea to subtract the mean from all data before even beginning any analysis that involves the summing of squares and products. In the present case, the choice of whether to perform such preprocessing is left to the user. It may not make much difference if the mean of the given values of $X$ is known to be small in magnitude relative to their standard deviation. Prior subtraction of the mean is advisable, in the absence of such knowledge.

\section{REFERENCES}

Emerson, P. L. A FORTRAN generator of polynomials orthonormal over unequally spaced and weighted abscissas. Educational and Psychological Measurement, 1965, 25, 867-871.

Emerson, P. L. Numerical construction of orthogonal polynomials from a general recurrence formula. Biometrics, 1968, 24, 695-701.

Forsythe, G. E. Generation and use of orthogonal polynomials for data fitting with a digital computer. Journal of the Society of Industrial Applied Mathematics, 1957, 5, 74-88.

GaIto, J. Unequal intervals and unequal $n$ in trend analysis. Psychological Bulletin, 1965, 63, 125-127.

Gaito, J., Turner, E. D. Error terms in trend analysis. Psychological Bulletin, 1963, 60, 464-474.

Grant, D. A. Analysis-of-variance tests in the analysis and comparison of curves. Psychological Bulletin, 1956, 53, 141-154.

House holden, A. S. Principles of numerical analysis. New York: McGraw-Hill, 1953.

Szegö, G. Orthogonal polynomials (Rev. ed.). Boston: SpauldingMoss, 1959. (Originally published, 1939.)

(Accepted for publication August 24, 1982.) 